Pacific Journal of Mathematics

POLYNOMIAL FORMS ON AFFINE MANIFOLDS

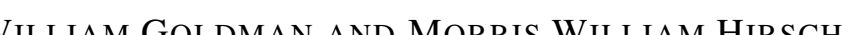




\section{POLYNOMIAL FORMS ON AFFINE MANIFOLDS}

\section{William M. Goldman aNd MoRris W. Hirsch}

An affine manifold is a differentiable manifold without boundary together with a maximal atlas of coordinate charts such that all coordinate changes extend to affine automorphisms of $\boldsymbol{R}^{n}$. These distinguished charts are called affine coordinate systems.

Throughout this paper $M$ denotes a connected affine manifold of dimension $n \geqq 1$. We write $E$ for $\boldsymbol{R}^{n}$.

A tensor (field) on $M$ is called polynomial if in all affine coordinate systems its coefficients are polynomial functions in $n$ variables. In particular a real-valued function on $M$ may be polynomial.

It is unknown whether there exists any compact affine manifold admitting a nonconstant polynomial function. The main purpose of this paper is to prove that for certain classes of affine manifolds there is no such function. These results are then applied to demonstrate that certain polynomial forms must also vanish. For related results, see Fried, Goldman, and Hirsch [2], Fried [1], [6], and [5].

1. Development, holonomy, and polynomial tensors. Let $p: \widetilde{M} \rightarrow M$ be a universal covering space. There is an immersion $D: \widetilde{M} \rightarrow E$, called the developing map, with the following properties (see e.g., [2]):

(1) $D$ is affine, i.e., in affine coordinates $D$ appears as an affine map; of $E$.

(2) $D$ is unique up to composition with an affine automorphism

We call $D(\tilde{M})$ the developing image.

Let $\pi$ denote the group of deck transformations of $\tilde{M}$. It follows from (2) that there is a homomorphism $\alpha: \pi \rightarrow \operatorname{Aff}(E)$, the group of affine automorphisms of $E$, such that $D$ is equivariant respecting $\alpha$, that is:

$$
D \circ g=\alpha(g) \circ D \text { for all } g \in \pi .
$$

We call $\alpha$ the affine holonomy. The composition

$$
\lambda: \pi \stackrel{\alpha}{\longrightarrow} \operatorname{Aff}(E) \stackrel{\beta}{\longrightarrow} G L(E),
$$

where $\beta$ is the natural homomorphism, is called the linear holonomy.

If $\alpha(\pi)$ fixes a point $p \in E$ then $M$ is called a radiant manifold. In this case we can compose $D$ with translation by $-p$ to obtain a new developing map whose corresponding affine holonomy fixes 
the origin. When $M$ is radiant we always assume $D$ is such that $\alpha(\pi)$ fixes the origin. Then the affine and linear holonomy coincide.

The simplest example of a radiant manifold is a Hopf manifold. Here $M$ is the quotient of $\boldsymbol{R}^{n}-\{0\}$ by the cyclic group generated by a linear expansion (a linear map all of whose eigenvalues have norm greater than one). One obtains more radiant manifolds by taking products of Hopf manifolds.

If $D: \widetilde{M} \rightarrow E$ is a homeomorphism then $M$ is said to be complete. In this case $M$ is the orbit space $E / \alpha(\pi)$.

Lemma 1. Suppose that $M$ admits an affine immersion $f: M \rightarrow E$. Then each polynomial tensor $T$ on $M$ is f-related to a unique polynomial tensor $S$ on $E$.

Proof. Let $f$ map a nonempty open set $U \subset M$ diffeomorphically onto an open set $V \subset E$. There is a unique tensor $S_{0}$ on $V$ which is $f$-related to $\left.T\right|_{U}$. Since $f$ is affine, $S_{0}$ is polynomial; hence $S_{0}$ extends to a unique polynomial tensor $S$ on $E$. The pullback $f^{*} S$ is a polynomial tensor on $M$ which agrees with $T$ in $U$; hence it equals $T$ because $M$ is connected. The uniqueness of $S$ is obvious.

Now let $T$ be a polynomial tensor on $M$ and let $\widetilde{T}=p^{*} T$ be the pullback of $T$ by $p: \widetilde{M} \rightarrow M$. Then $\widetilde{T}$ is a polynomial tensor on $\widetilde{M}$, where $\widetilde{M}$ is given the affine structure induced from $M$ by $p$. Since the developing map $D: \widetilde{M} \rightarrow E$ is an affine immersion, Lemma 1 implies that $\widetilde{T}$ is $D$-related to a unique polynomial tensor $S$ on $E$. The equivariance of $D$ and the fact that $\widetilde{T}$ is $\pi$-invariant shows that $S$ is $\alpha(\pi)$-invariant.

Conversely, starting with an $\alpha(\pi)$-invariant polynomial tensor $S$ on $E$, there is a unique polynomial tensor $T$ on $M$ such that $p^{*} T$ is $D$-related to $S$.

We have established a natural one-to-one correspondence between polynomial tensors on $M$ and $\alpha(\pi)$-invariant polynomial tensors on $E$.

2. Polynomial functions. Let $D: \widetilde{M} \rightarrow E$ be the developing map of the affine manifold $M$. Let $\Gamma=\alpha(\pi) \subset$ Aff $(E)$ be the affine holonomy group of $M$.

Let $f: M \rightarrow R$ be a polynomial function. In $\S 1$ we showed that there is a unique polynomial map $g: E \rightarrow R$ such that the following diagram commutes:

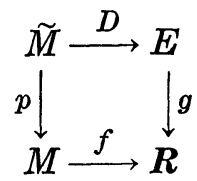


and $g$ is $\Gamma$-invariant.

Suppose $f$ is bounded on $M$. Then clearly $g$ is bounded on the developing image $D(\widetilde{M})$. It follows that if $L \subset E$ is a line such that $L \cap D(\widetilde{M})$ is unbounded, then $\left.g\right|_{L}$ is constant. This implies that $g$, and hence also $f$, is constant if $M$ is compact and complete. More generally:

THEOREM 2.1. If $D(\widetilde{M})$ contains a nonempty open cone then every bounded polynomial function on $M$. is constant.

Proof. Let $C \subset D(\widetilde{M})$ be the open cone, with vertex $q \in E$. For every $x \in C$ the line $x q$ meets $C$, and hence $D(\widetilde{M})$, in a half line. Therefore $g$ is constant on $x q$. Since $C$ is open, the set of vertices $q^{\prime}$ of open cones $C^{\prime}$ contained in $C$ is open. It follows that $g$ is constant on a nonempty open set. Thus $g$, and also $f$, is constant.

TheOREM 2.2. Let $D(\widetilde{M}) \supset H(C)$ where $C \subset E$ is a nonempty open cone and $H: E \rightarrow E$ is a polynomial map whose Jacobian is not identically zero. Then every bounded polynomial function on $M$ is constant.

Proof. As in the proof of 2.1, one sees that $g \circ H: C \rightarrow E$ is constant. It follows that $g \circ H$ is constant. Since $H$ has nonzero Jacobian at some point, the inverse function theorem implies that $g$ is constant on an open set. Hence $g$ and $f$ are constant.

In Goldman [4] there is an example of a nonradiant compact affine 3-manifold with solvable fundamental group whose developing image is the convex set $\left\{(x, y, z) \in \boldsymbol{R}^{3}: y>x^{2}\right\}$. This set contains no open cone, but is equivalent to an open half-space by a polynomial automorphism of $\boldsymbol{R}^{3}$.

THEOREM 2.3. Every polynomial function on a compact radiant manifold $M$ is constant.

Proof. We may assume the affine holonomy group $\Gamma$ is contained in $G L(E)$. Therefore it preserves the vector field on $E$ defined by the identity map $I(x)=x$, for $x \in E$. We consider $I$ as a $\Gamma$-invariant tensor; therefore $I$ corresponds to a polynomial vector field $R$ on $M$ called the radiant vector field.

Let $\left\{\phi_{t}\right\}_{t \in R}$ be the flow of $R$. Let $\widetilde{R}=p^{*} R$ be the radiant vector field of $\tilde{M}$. Let $\tilde{\phi}_{t}$ be the flow of $\widetilde{R}$. Then $D: \widetilde{M} \rightarrow E$ is equivariant respecting $\tilde{\phi}_{t}$ and the flow of $I$, which is $x \rightarrow e^{t} x$. Therefore $D(\tilde{M})$ is invariant under multiplication by positive scalars, and hence an 
open cone. Thus 2.2 implies 2.3.

Let $A(\Gamma) \subset$ Aff $(E)$ be the smallest algebraic subgroup of Aff $(E)$ containing $\Gamma$. We think of $A(\Gamma)$ as acting on $E$ by affine automorphisms.

THEOREM 2.4. Suppose that $A(\Gamma)$ has an open orbit in $E$. Then every polynomial function on $M$ is constant.

Proof. Obvious, since $\Gamma$-invariant implies $A(\Gamma)$-invariant.

A condition generalizing radiance is decomposability: We say that $\Gamma \subset \operatorname{Aff}(E)$ preserves a decomposition of $E$ if there is a directsum decomposition $E=E_{1} \oplus F$, where $E_{1}$ is $\Gamma$-invariant and $F$ is $\beta(\Gamma)$-invariant, where $\beta$ : Aff $(E) \rightarrow G L(E)$ is the natural homomorphism. If $M$ has decomposable holonomy, then the radiant vector field on $F$ defines a $C^{\infty}$ vector field $R_{F}$ as in $\S 6$ of [2]. The sets $D^{-1}(a \times F), a \in E$, are leaves of a foliation $\hat{\mathscr{F}}$ of $\tilde{M}$. Being $\pi$ invariant, $\hat{\mathscr{F}}$ comes from a foliation $\mathscr{F}$ of $M$. Each leaf $L$ of $\mathscr{F}$, in its manifold topology, has a natural affine structure, which is radiant, having $R_{F}$ as its radiant vector field.

THEOREM 2.5. If $M$ is compact, has decomposable holonomy as above, and $\operatorname{dim} E_{1}=1$, then every polynomial function on $M$ is constant.

Proof. Since $M$ is compact, the $F$-radiant vector field $R_{F}$ on $M$ is integrable; it then follows from 2.1 that on each leaf of $\mathscr{F}$ every polynomial function $f: M \rightarrow \boldsymbol{R}$ is constant. Then the corresponding polynomial $g: E_{1} \oplus F \rightarrow \boldsymbol{R}$ is constant on the cosets of $F$ and $g$ factors through a polynomial $h$ as

$$
E^{\mathrm{t}} \oplus F \longrightarrow E_{1} \stackrel{h}{\longrightarrow} \boldsymbol{R},
$$

that is, $g(x, y)=h(x)$.

Since $g$ is $\Gamma$-invariant, $h$ is invariant under the affine action of $\Gamma$ on $E_{1}$. Passing to a double covering of $M$ we may assume that $\Gamma$ preserves orientation on $E_{1}$. Now $E_{1} \cong \boldsymbol{R}$, so every $\gamma \in \Gamma$ which acts nontrivially on $E_{1}$ has infinite orbit. But a polynomial invariant under such a $\gamma$ is constant. Hence either $\left.g\right|_{E_{1}}$ is constant, forcing $g$ and $f$ to be constant, or else $\Gamma$ acts trivially on $E_{1}$. But then $\Gamma$ fixes the origin of $E$, so $M$ is radiant and the theorem follows from 2.3 .

One important case of decomposable holonomy occurs when $M$ 
is compact and $\Gamma$ is a nilpotent group. Then the holonomy of $M$ decomposes $E=E_{1} \oplus F$, in such a way that under the projection $E \rightarrow E_{1}$ the developing image $D(\tilde{M})$ is a fibration onto $E$ (see [2], 6.8). It then follows (see also [1]):

THEOREM 2.6. A compact affine manifold whose affine holonomy group contains a nilpotent subgroup of finite index cannot admit a nonconstant polynomial function.

Another proof of 2.6 follows from the fact that if $M$ is a compact affine manifold with nilpotent affine holonomy group $\Gamma$, then either $E_{1}=E$ and $M$ is complete or else every $\Gamma$-invariant algebraic subset of $E$ contains $E_{1}$ [6]. Since the level sets of an invariant polynomial $g: E \rightarrow R$ are $\Gamma$-invariant and disjoint, this contradiction proves 2.6 .

In a forthcoming paper [1], D. Fried proves that on all compact affine manifolds of dimension three there are no nonconstant polynomial functions.

In [6] we prove that on all compact affine manifolds with "parallel volume," i.e., the affine structure is volume-preserving, there are no nonconstant polynomial functions.

3. Polynomial symmetric and exterior forms, A polynomial tensor $T$ on an affine manifold has a well-defined degree. If the degree is 0 we call the tensor parallel. There is then a constant tensor $S$ on $E$ such that in every affine chart the components of $T$ appear as $S$.

A parallel symmetric $k$-form on $M$ corresponds to a $\Gamma$-invariant symmetric $k$-linear map $E \times \cdots \times E \rightarrow \boldsymbol{R}$.

THEOREM 3.1. On a compact radiant manifold every parallel symmetric $k$-form $(k \geqq 1)$ is zero.

Proof. Let $T$ be the symmetric $k$-form and $R$ the radiant vector field. Define a function $f: M \rightarrow R$ by $f(x)=T(R(x), \cdots, R(x))$. Then $f$ is a polynomial function; by $2.3 f$ is constant.

Let $S: E^{k} \rightarrow \boldsymbol{R}$ be the symmetric $k$-linear map corresponding to $T$. The radiant vector field corresponds to the identity vector field. Consider the map $g: E \rightarrow \boldsymbol{R}, g(x)=S(x, \cdots, x)$. Since $g$ is a polynomial function on $E$ which corresponds to $f$, we see that $g$ is constant. But the $k$ th derivative of $g$ at 0 , considered as a symmetric $k$-linear map, equals $k ! S$. Hence $S=0$. 
THEOREM 3.2. On a compact radiant manifold every polynomial closed 1-form is zero.

Proof. Let $\omega$ be such a 1-form and let $R$ be the radiant vector field. Then $\omega(R)$ is a polynomial function, hence constant by 2.3.

The corresponding closed 1 -form $\omega^{\prime}$ on $E$ is exact; thus $\omega^{\prime}=d u$ where $u: E \rightarrow R$ is a polynomial function. Now $\omega^{\prime}=\Sigma\left(\partial u / \partial x_{i}\right) d x_{i}$; so the constancy of $\omega^{\prime}\left(R^{\prime}\right)$ means that $\Sigma x_{i}\left(\partial u / \partial x_{i}\right)$ is constant. Since it vanishes at 0 , it must vanish identically.

This says the derivative of $u$ along any ray through the origin vanishes identically. Thus $u$ is constant along such rays; so $u(x)=$ $u(0)$ for all $x$. This shows $\omega^{\prime}=d u=0$; hence $\omega=0$.

Theorem 3.2 cannot be extended to polynomial 1-forms which are not closed, nor does it extend to nonradiant compact affine manifolds. In [2], $\S 6$ there is an example of a compact radiant 3-manifold $M$ which admits a parallel exterior 2-form $\omega$. There are linear coordinates in $R^{3}$ so that $R=x(\partial / \partial x)+y(\partial / \partial y)+z(\partial / \partial z)$ is the radiant vector field and $\omega=d x \wedge d y$. The interior product $\iota_{R} \omega=$ $x d y-y d x$ is a nonzero polynomial 1 -form on $M$.

In general for an affine manifold $M$ we may consider the polynomial de Rham complex $A_{\mathrm{poly}}^{*}(M)$ consisting of all polynomial exterior differential forms on $M$, and form its cohomology $H_{\text {poly }}^{*}(M)$, the polynomial de Rham cohomology of $M$. There is a map $H_{\mathrm{poly}}^{*}(M) \rightarrow$ $H^{*}(M ; \boldsymbol{R})$ induced by the inclusion $A_{\mathrm{poly}}^{*}(M) \rightarrow A^{*}(M)$ of chain complexes.

Conjecture 3.3. If $M$ is a complete affine manifold, then the natural mapping $H_{\mathrm{poly}}^{*}(M) \rightarrow H^{*}(M ; \boldsymbol{R})$ is an isomorphism.

In [2], $\S 8$ this conjecture is proved when $M$ is compact and $\pi_{1}(M)$ is nilpotent. In [3] this is extended to $M$ compact and $\pi_{1}(M)$ virtually solvable.

In contrast to the complete case, we have the following result:

THEOREM 3.4. Let $M$ be a radiant affine manifold. Then for $k>0, H_{\mathrm{poly}}^{k}(M)=0$, i.e., every closed polynomial $k$-form is exact.

Proof. Let $\omega$ be a polynomial exterior $k$-form on $M$. Let $\omega^{\prime}$ denote the corresponding form on $E$. Decompose $\omega^{\prime}$ into its components $\omega^{\prime}=\omega_{m}^{\prime}+\cdots+\omega_{1}^{\prime}+\omega_{0}^{\prime}$ where $\omega_{p}^{\prime}$ is an exterior $k$-form whose coefficients are homogeneous polynomials of degree $p$. Since $M$ is radiant each $\omega_{p}^{\prime}$ defines a $k$-form $\omega_{p}$ invariantly on $M$. It is 
easy to see that $\omega$ is closed if and only if each $\omega_{p}$ is closed. Hence to prove the theorem it suffices to assume that the coefficients of $\omega$ are homogeneous polynomials are some degree $m$.

Let $R^{\prime}$ (resp. $R$ ) denote the radiant vector field on $E$ (resp. $M$ ) and let $\xi_{t}^{\prime}$ (resp. $\xi_{t}$ ) be the corresponding flow. It is easy to see that $\xi_{t}^{*} \omega=e^{(k+m) t_{\omega}}$ so we may take the Lie derivative $\mathscr{L}_{R} \omega=(k+m) \omega$. Now $\omega=(k+m)^{-1} \mathscr{L}_{R} \omega=d\left((k+m)^{-1} \iota_{R} \omega\right)$ is exact.

In a special case, there is a converseto 3.4 :

THEOREM 3.5. Let $M$ be a compact affine manifold whose holonomy group is nilpotent. If $H_{\mathrm{poly}}^{k}(M)=0$ for all $k>0$, then $M$ is radiant.

Proof. It is proved in [7] (see [6] for another application) that unless $M$ is radiant there is a parallel exterior $k$-form $\omega$ with nonzero cohomology class $[\omega] \in H^{k}(M)$. Since $\omega$ is parallel, it defines a cohomology class $\{\omega\} \in H_{\mathrm{poly}}^{k}(M)$. Since the image of $\{\omega\}$ in $H^{k}(M)$ is nonzero, $\{\omega\}$ defines a nonzero element of $H_{\mathrm{poly}}^{k}(M)$.

\section{REFERENCES}

1. D. Fried, Polynomials on affine manifolds, (to appear in Comment. Math. Helv.).

2. D. Fried, W. Goldman, and M. Hirsch, Affine manifolds with nilpotent holonomy, Comm. Math. Helv., 56 (1981), 487-523.

3. W. Goldman, On the polynomial cohomology of affine manifolds, (in preparation).

4. - Two examples of affine manitolds, (to appear in Pacific J. Math.).

5. - Rational functions on affine 3-manifolds, (in preparation).

6. W. Goldman and M. Hirsch, Parallel characteristic classes of affine manifolds, (in preparation).

7. W. Goldman, M. Hirsch, and G. Levitt, Invariant measures for affine foliations, Technical report 34, Center for Pure and Applied Math., U. California at Berkeley, 1980 ,

Received June 10, 1981.

University of Colorado

BOULDER, CO 80309

AND

UNIVERSity OF CALIForNiA

Berkeley, CA 94720 



\section{PACIFIC JOURNAL OF MATHEMATICS}

\section{EDITORS}

DONALD BABBITT (Managing Editor)

J. DUGUNDJI

University of California

Los Angeles, California 90024

Hugo Rossi

University of Utah

Salt Lake City, UT 84112

C. C. Moore and Arthur Agus

Department of Mathematics

University of Southern California

Los Angeles, California 90007

R. FinN and J. Milgram

Stanford University

Stanford, California 94305

University of California

Berkeley, CA 94720

\section{ASSOCIATE EDITORS}
R. ARNES
E. F. BeCKenBaCH
B. H. NEUMANN
F. WoLF
K. YoshidA

\section{SUPPORTING INSTITUTIONS}

UNIVERSITY OF ARIZONA

UNIVERSITY OF BRITISH COLUMBIA

CALIFORNIA INSTITUTE OF TECHNOLOGY

UNIVERSITY OF CALIFORNIA

MONTANA STATE UNIVERSITY

UNIVERSITY OF NEVADA, RENO

NEW MEXICO STATE UNIVERSITY

OREGON STATE UNIVERSITY
UNIVERSITY OF OREGON

UNIVERSITY OF SOUTHERN CALIFORNIA

STANFORD UNIVERSITY

UNIVERSITY OF HAWAII

UNIVERSITY OF TOKYO

UNIVERSITY OF UTAH

WASHINGTON STATE UNIVERSITY

UNIVERSITY OF WASHINGTON 


\section{Pacific Journal of Mathematics}

\section{Vol. 101, No. $1 \quad$ November, 1982}

Natália Bebiano, On the evaluation of permanents $\ldots \ldots \ldots \ldots \ldots \ldots \ldots \ldots$

David Borwein and Bruce Brigham Watson, Tauberian theorems between

the logarithmic and Abel-type summability methods $\ldots \ldots \ldots \ldots \ldots \ldots 11$

Leo George Chouinard, II, Hermite semigroup rings $\ldots \ldots \ldots \ldots \ldots \ldots \ldots$

Kun-Jen Chung, Remarks on nonlinear contractions $\ldots \ldots \ldots \ldots \ldots \ldots . \ldots 4$

Lawrence Jay Corwin, Representations of division algebras over local

fields. II ......................................... 49

Mahlon M. Day, Left thick to left lumpy—a guided tour $\ldots \ldots \ldots \ldots \ldots 71$

M. Edelstein and Mo Tak Kiang, On ultimately nonexpansive

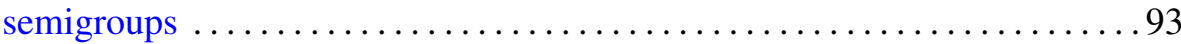

Mary Rodriguez Embry, Semigroups of quasinormal operators . ........ 103

William Goldman and Morris William Hirsch, Polynomial forms on

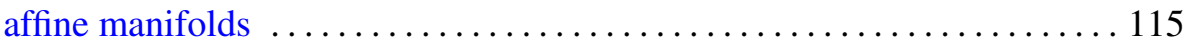

S. Janakiraman and T. Soundararajan, Totally bounded group topologies

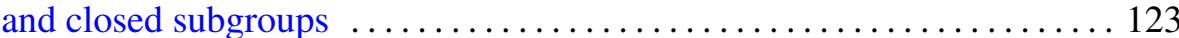

John Rowlay Martin, Lex Gerard Oversteegen and Edward D.

Tymchatyn, Fixed point set of products and cones $\ldots \ldots \ldots \ldots \ldots \ldots 133$

Jan van Mill, A homogeneous Eberlein compact space which is not metrizable ........................................ 141

Steven Paul Plotnick, Embedding homology 3-spheres in $S^{5} \ldots \ldots \ldots \ldots 147$

Norbert Riedel, Classification of the $C^{*}$-algebras associated with minimal rotations

Benedict Seifert, Combinatorial and geometric properties of weight systems of irreducible finite-dimensional representations of simple split Lie algebras over fields of 0 characteristic

James E. Simpson, Dilations on locally convex spaces

Paolo M. Soardi, Schauder bases and fixed points of nonexpansive mappings

Yoshio Tanaka, Point-countable $k$-systems and products of $k$-spaces

Fausto A. Toranzos, The points of local nonconvexity of starshaped sets . . . 209

Lorenzo Traldi, The determinantal ideals of link modules. I . . . . . . . . 215

P. C. Trombi, Invariant harmonic analysis on split rank one groups with applications

Shinji Yamashita, Nonnormal Blaschke quotients 